\title{
Correction to: The Medial Septum Is Insulin Resistant in the AD Presymptomatic Phase: Rescue by Nerve Growth Factor-Driven IRS 1 Activation
}

\author{
Valentina Sposato ${ }^{1}$ - Nadia Canu ${ }^{2,3}$. Elena Fico ${ }^{2,4}$ - Salvatore Fusco ${ }^{5}$ - Giulia Bolasco ${ }^{6}$. Maria Teresa Ciotti ${ }^{1}$. \\ Matteo Spinelli ${ }^{5}$. Delio Mercanti ${ }^{2}$. Claudio Grassi ${ }^{5,7}$ • Viviana Triaca ${ }^{2}$ (D) $\cdot$ Pietro Calissano ${ }^{1,2}$
}

Published online: 21 February 2019

(C) Springer Science+Business Media, LLC, part of Springer Nature 2019

\section{Correction to: Mol Neurobiol (2019) 56(1): 535-552 https://doi.org/10.1007/s12035-018-1038-4}

The authors, due to the change in one of the University name at the affiliation, hereby correct the proof.

Affiliation 7 should now be presented as "Fondazione Policlinico Universitario A. Gemelli IRCCS, Rome, Italy" instead of "Fondazione Policlinico Universitario Agostino Gemelli, Rome, Italy".

Publisher's Note Springer Nature remains neutral with regard to jurisdictional claims in published maps and institutional affiliations.

The online version of the original article can be found at https://doi.org/ $10.1007 / \mathrm{s} 12035-018-1038-4$

\author{
Viviana Triaca \\ viviana.triaca@ibcn.cnr.it \\ Valentina Sposato \\ v.sposato@ebri.it \\ Nadia Canu \\ n.canu@inmm.cnr.it \\ Elena Fico \\ fico_elena@yahoo.it \\ Salvatore Fusco \\ salvatore.fusco@unicatt.it \\ Giulia Bolasco \\ giulia.bolasco@embl.it \\ Maria Teresa Ciotti \\ teresa.ciotti@inmm.cnr.it \\ Matteo Spinelli \\ matteo.spinelli@unicatt.it \\ Delio Mercanti \\ delio.mercanti@cnr.it
}

Claudio Grassi
claudio.grassi@unicatt.it

Pietro Calissano

pietro.calissano@inmm.cnr.it

European Brain Research Institute (EBRI) Rita Levi-Montalcini Foundation, Viale Regina Elena 295, Rome, Italy

2 National Research Council (CNR), Institute of Cell Biology and Neurobiology, Via del Fosso di Fiorano 64, Rome, Italy

3 Department of System Medicine, Section of Physiology, University of Rome "TorVergata", Rome, Italy

4 Department of Biotechnological and Applied Clinical Sciences, University of L'Aquila, L'Aquila, Italy

5 Institute of Human Physiology, Università Cattolica del Sacro Cuore, Rome, Italy

6 European Molecular Biology Laboratory (EMBL), Monterotondo Outstation, Rome, Italy

7 Fondazione Policlinico Universitario A. Gemelli IRCCS, Rome, Italy 